\title{
COMPACT MEANS IN THE PLANE
}

\author{
PHILIP BACON
}

1. Results. An $n$-mean $(n \geqq 2)$ is a nonvoid Hausdorff space $X$ together with a continuous symmetric idempotent function (which is also called an $n$-mean) from $X^{n}$ into $X$. A space on which an $n$-mean can be defined is called an $m_{n}$-space $[2$, p. 210]. In the present note we show that a compact $m_{n}$-space embedded in the cartesian plane $R^{2}$ does not separate $R^{2}$ and, as a partial converse, that any compact locally connected subset of $R^{2}$ that does not separate $R^{2}$ is an $m_{n}$ space.

These two theorems reduce the problem of characterizing compact $m_{n}$-spaces in $R^{2}$ to the question: When is a nonvoid compact nonlocally connected subset of $R^{2}$ with connected complement an $m_{n}$ space? The question is not answered here, but the answer cannot be either "always" or "never," for, on the one hand, there is a compact subset of $R^{2}$ with connected complement that is not an $m_{2}$-space [3] and, on the other hand, a semilattice described in [1, p. 185, Example 1 ] is a 2 -mean on a compact connected subset of $R^{2}$ that is not locally connected.

2. Proofs. Our lemmas concern Cech homology theory on the category of compact pairs. We establish our notation with the following remarks.

Suppose $G$ is an abelian group, $X$ is a finite complex and $A$ is a subcomplex of $X$. The $G$-valued $n$-chains of the oriented $n$-simplexes of $X$ that assume the value 0 on the oriented $n$-simplexes of $A$ form, under functional addition, an abelian group which will be denoted by $C_{n}(X, A ; G)$. The boundary operator $\partial$ is defined in the usual manner $[7$, p. 111] and

$Z_{n}(X, A ; G)$ is the kernel of $\partial: C_{n}(X, A ; G) \rightarrow C_{n-1}(X, A ; G)$;

$B_{n}(X, A ; G)$ is the image of $\partial: C_{n+1}(X, A ; G) \rightarrow C_{n}(X, A ; G)$; and

$H_{n}(X, A ; G)=Z_{n}(X, A ; G) / B_{n}(X, A ; G)$.

For each integer $n$, a simplicial map $f:(X, A) \rightarrow(Y, B)$ induces a homomorphism $C_{n}(f): C_{n}(X, A ; G) \rightarrow C_{n}(Y, B ; G)$ which in turn induces a homomorphism $H_{n}(f): H_{n}(X, A ; G) \rightarrow H_{n}(Y, B ; G)$. If $X$ is a compact Hausdorff space, $\operatorname{Cov}(X)$ is the set of all finite open covers of $X$. If $K$ is in $\operatorname{Cov}(X), X_{K}$ is the nerve of $K$. If $(X, A)$ is a compact pair (that is, $X$ is compact and Hausdorff and $A$ is a closed subset of $X)$, if $K, J \in \operatorname{Cov}(X)$ and if $J$ refines $K$, there is a unique projection

Received by the editors October 1, 1968. 


$$
\pi(K, J): H_{n}\left(X_{J}, A_{J} ; G\right) \rightarrow H_{n}\left(X_{K}, A_{K} ; G\right) .
$$

The inverse limit group defined by all such projections is the $n$th Cech homology group for $(X, A)$ with coefficients in $G$ and is denoted by $H_{n}(X, A ; G)$. If $h$ is in $H_{n}(X, A ; G)$ and if $K$ is in $\operatorname{Cov}(X)$, the $K$ th coordinate of $h$ is $h_{K}$. A continuous map $f:(X, A) \rightarrow(Y, B)$ between compact pairs induces a homomorphism $f_{*}: H_{n}(X, A ; G)$ $\rightarrow H_{n}(Y, B ; G)$.

(2.1) Suppose $X$ is a connected compact Hausdorff space, $p \in X$ and $n$ is an integer $\geqq 2$. Let maps $d^{n}, f^{n j}: X \rightarrow X^{n}(j \in\{1, \cdots, n\})$ be defined by the formulas

$$
\begin{aligned}
\left(d^{n} x\right)_{i} & =x ; & \\
\left(f^{n j} x\right)_{i} & =x, & i=j, \\
& =p, & i \neq j ;
\end{aligned}
$$

$x \in X, i \in\{1, \cdots, n\}$. If $G$ is an abelian group and $h \in H_{1}(X ; G)$, then $d_{*}^{n} h=\sum_{j=1}^{n} f_{*}^{n j} h$.

Proof. We first consider the case $n=2$. Suppose $E \in \operatorname{Cov}\left(X^{2}\right)$. Since $X$ is compact, there is a $J$ in $\operatorname{Cov}(X)$ such that

$$
K=\{U \times V: U, V \in J\}
$$

refines $E$. Let $P$ be a member of $J$ that contains $p$. Define simplicial maps

$$
F^{21}, F^{22}, D^{2}: X_{J} \rightarrow\left(X^{2}\right)_{K}
$$

by the rules $F^{21} U=U \times P, F^{22} U=P \times U$ and $D^{2} U=U \times U$ for all $U$ in $J$. Observe that $f^{21} U \subset F^{21} U, f^{22} U \subset F^{22} U$ and $d^{2} U \subset D^{2} U$ whenever $U$ is in $J$. Suppose $g \in G, m \geqq 3$ and $z$ in $Z_{1}\left(X_{J} ; G\right)$ is such that

$$
z=g \sum_{i=1}^{m} V_{i-1} V_{i}
$$

where $V_{0}=V_{m}=P$ and $V_{i-1} \neq V_{i}$ whenever $i \in\{1, \cdots, m\}$. Define $w \in C_{2}\left(\left(X^{2}\right)_{K} ; G\right)$ by

$$
\begin{aligned}
w=g[ & \sum_{i=1}^{n-1} \sum_{j=1}^{i}\left(V_{i} \times V_{j}\right)\left(V_{i+1} \times V_{j}\right)\left(V_{i+1} \times V_{j+1}\right) \\
& \left.+\sum_{i=2}^{n-1} \sum_{j=1}^{i-1}\left(V_{i} \times V_{j}\right)\left(V_{i+1} \times V_{j+1}\right)\left(V_{i} \times V_{j+1}\right)\right] .
\end{aligned}
$$

Then direct computation shows that 


$$
C_{1}\left(F^{21}\right)(z)+C_{1}\left(F^{22}\right)(z)-C_{1}\left(D^{2}\right)(z)=\partial w \text {. }
$$

Since $X_{J}$ is connected, every cycle in $Z_{1}\left(X_{J} ; G\right)$ is a finite sum of cycles of form (1). Hence for any $z$ in $Z_{1}\left(X_{J} ; G\right)$ there is a $w$ in $C_{2}\left(\left(X^{2}\right)_{K} ; G\right)$ such that (2) holds. It follows immediately that, if $h \in H_{1}\left(X_{J} ; G\right)$,

$$
H_{1}\left(F^{21}\right)(h)+H_{1}\left(F^{22}\right)(h)-H_{1}\left(D^{2}\right)(h)=0 .
$$

Now suppose $h \in H_{1}(X ; G)$. Then

$$
\begin{aligned}
\left(f_{*}^{21} h+f_{*}^{22} h-d_{*}^{2} h\right)_{E} & =\pi(E, K)\left[\left(f_{*}^{21} h\right)_{K}+\left(f_{*}^{22} h\right)_{K}-\left(d_{*}^{2} h\right)_{K}\right] \\
& =\pi(E, K)\left[H_{1}\left(F^{21}\right)\left(h_{J}\right)+H_{1}\left(F^{22}\right)\left(h_{J}\right)-H_{1}\left(D^{2}\right)\left(h_{J}\right)\right] \\
& =\pi(E, K)(0)=0,
\end{aligned}
$$

which completes the proof for the case $n=2$.

The proof for $n>2$ proceeds by an induction on $n$. Let $h$ be in $H_{1}(X ; G)$ and define maps $k: X^{n} \rightarrow X^{n+1}$ and $g: X^{2} \rightarrow X^{n+1}$ by the formulas

$$
\begin{aligned}
k\left(x_{1}, \cdots, x_{n}\right) & =\left(x_{1}, \cdots, x_{n}, x_{n}\right) ; \\
g\left(x_{1}, x_{2}\right) & =\left(p, \cdots, p, x_{1}, x_{2}\right) .
\end{aligned}
$$

Using the case $n=2$ we have $\left(k f^{n n}\right)_{* h}=\left(g d^{2}\right)_{* h}=\left(g f^{21}\right)_{*} h+\left(g f^{22}\right)_{* h}$ $=f_{*}^{n+1, n} h+f_{*}^{n+1, n+1} h$. Using this and the inductive hypothesis we have $d_{*}^{n+1} h=\left(k d^{n}\right)_{*} h=\sum_{j=1}^{n-1}\left(k f^{n j}\right)_{*} h+\left(k f^{n n}\right)_{*} h=\sum_{j=1}^{n+1}\left(k f^{n j}\right)_{*} h$.

Throughout the remainder of this paper $\boldsymbol{Z}_{n}$ denotes a cyclic group of order $n$.

(2.2) Suppose $X$ is a compact connected Hausdorff space, $p \in X, n$ is an integer $\geqq 2$ and $m: X^{n} \rightarrow X$ is a continuous function such that if $x \in X$,

(1) $m(x, x, \cdots, x)=x$;

(2) $m(x, p, \cdots, p)=m(p, x, p, \cdots, p)=\cdots=m(p, \cdots, p, x)$. Then $H_{1}\left(X ; Z_{n}\right)=0$.

Proof. We suppose the hypotheses of (2.2) and define $f^{n j}, d^{n}$ : $X \rightarrow X^{n}$ as in the hypothesis of (2.1). Observe that $m d^{n}$ is the identity on $X$ and that, for $j \in\{2, \cdots, n\}, m f^{n j}=m f^{n 1}$. Let $h$ be in $H_{1}\left(X ; Z_{n}\right)$. By (2.1), $h=m_{*} d_{*}^{n} h=\sum_{j=1}^{n} m_{*} f_{*}^{n j} h=n\left(m_{*} f_{*}^{n 1} h\right)=0$, the last equality holding because the use of $\boldsymbol{Z}_{n}$ as coefficient group insures that every element of a Cech group is of order $n$.

(2.3) Suppose $n \geqq 2, X$ is a compact subset of $\boldsymbol{R}^{n}$ and $G$ is a nontrivial abelian group for which $H_{n-1}(X ; G)=0$. If the Cech homology theory 
on compact pairs using $G$ as coefficient group is exact, then $R^{n}-X$ is connected.

Proof. Suppose on the contrary $R^{n}-X$ has a bounded component $C$. Let $E$ be an $n$-ball with $X \cup C$ in its interior and its bounding $(n-1)$-sphere denoted by $S$. Define $T=E-C$. Since $T$ is a proper subset of $E$ and contains $S, S$ is a retract of $T$. Accordingly the inclusion map $k: S \subset T$ induces a monomorphism $k_{*}: H_{n-1}(S ; G) \rightarrow H_{n-1}$ $(T ; G)$ and since $H_{n-1}(S ; G) \approx G \neq 0, H_{n-1}(T ; G) \neq 0$. In the following commutative diagram the dimension preserving homomorphisms are induced by appropriate inclusion maps.

$$
\begin{array}{r}
H_{n-1}(X ; G) \stackrel{\partial_{1}}{\leftarrow} H_{n}(X \cup C, X ; G) \\
\downarrow v_{*} \quad \stackrel{\partial_{2}}{\longleftarrow} H_{n}(E, T ; G) . \\
H_{n-1}(E ; G) \stackrel{j_{*}}{\leftarrow} H_{n-1}(T ; G) \stackrel{ }{\leftarrow} H_{n}
\end{array}
$$

Since $H_{n-1}(X ; G)=0, \partial_{2} u_{*}=v_{*} \partial_{1}=0$. By $[6$, p. 266, Theorem 5.4], the excision $u_{*}$ is an isomorphism. Hence $\partial_{2}$ is a 0 -homomorphism and, by exactness, $j_{*}$ is a monomorphism. Since $H_{n-1}(T ; G) \neq 0, H_{n-1}(E ; G) \neq 0$, which is false.

(2.4) If a compact subset $X$ of $R^{2}$ is an $m_{n}$-space, $R^{2}-X$ is connected.

Proof. Suppose, on the contrary, that a compact subset $X$ of $R^{2}$ admits an $n$-mean $m$ and separates a point $p$ from a point $q . R^{\mathbf{2}}$ is locally connected and unicoherent $[5$, p. 73, Corollary 6]. By a theorem of A. H. Stone [8, p. 429, Theorem 1] some component $C$ of $X$ separates $p$ from $q$. The restriction of $m$ to $C^{n}$ is an $n$-mean on $C$ [2, p. 212, Satz 4]. By (2.2) $H_{1}\left(C, Z_{n}\right)=0$. The Cech homology theory for compact pairs with $\boldsymbol{Z}_{n}$ as coefficient group is exact $[6$, p. 248, Theorem 7.6]. By (2.3), $R^{2}-C$ is connected, which is a contradiction.

(2.5) A nonvoid compact locally connected subset of $R^{2}$ that does not separate $R^{2}$ is an $m_{n}$-space.

Proof. Suppose $X$ is a nonvoid compact locally connected subset of $R^{2}$ and $R^{2}-X$ is connected. Consider first the case in which $X$ is connected. The function $m:\left(R^{2}\right)^{n} \rightarrow R^{2}$ defined by $m\left(p_{1}, \cdots, p_{n}\right)$ $=(1 / n) \sum_{i=1}^{n} p_{i}$ shows that $R^{2}$ is an $m_{n}$-space. Since $X$ is a retract of $R^{2}[4, \mathrm{p} .132,(13.1)]$ and any retract of an $m_{n}$-space is an $m_{n}$-space [2, p. 212, Satz 3], $X$ is an $m_{n}$-space.

If $X$ is not connected, $X$ has at most finitely many components, $C_{1}, \cdots, C_{k}$, each of which, by the above argument, is an $m_{n}$-space. For each $i$ in $\{1, \cdots, k\}$ let $m_{i}: C_{i}^{n} \rightarrow C_{i}$ be an $n$-mean. Let $p$ be a 
point of $X$. We define an $n$-mean $m: X^{n} \rightarrow X$ by the rule

$$
\begin{aligned}
m q & =m_{i} q, \quad \text { if } q \in C_{i}^{n}, \quad i \in\{1, \cdots, k\} ; \\
& =p, \quad \text { if } q \in X^{n}-\bigcup_{i=1}^{k} C_{i}^{n} .
\end{aligned}
$$

\section{REFERENCES}

1. L. W. Anderson and L. E. Ward, Jr., One-dimensional topological semilattices, Illinois J. Math. 5 (1961), 182-186.

2. G. Aumann, Über Räume mit Mittelbildungen, Math. Ann. 119 (1943), 210-215.

3. P. Bacon, An acyclic continuum that admits no mean, Fund. Math. (to appear).

4. K. Borsuk, Theory of retracts, PWN-Polish Sci. Publ., Warsaw, 1967.

5. S. Eilenberg, Transformations continues en circonférence et la topologie du plan, Fund. Math. 26 (1936), 61-112.

6. S. Eilenberg and N. Steenrod, Foundations of algebraic topology, Princeton Univ. Press, Princeton, N. J., 1952.

7. W. Hurewicz and H. Wallman, Dimension theory, Princeton Univ. Press, Princeton, N. J., 1941.

8. A. H. Stone, Incidence relations in unicoherent spaces, Trans. Amer. Math. Soc. 65 (1949), 427-447.

UNIVERSITY OF FLORIDA 